\title{
FitoestimulaçÃo POR Stizolobium aterrimum como PROCESSO DE REMEdiação de Solo ConTAMinado COM TRIFloXYSUlfuron- SODIUM $^{1}$
}

\author{
Phyto-Stimulation by Stizolobium aterrimum as Remediation of Soil Contaminated with \\ Trifloxysulfuron-Sodium
}

\author{
SANTOS, E.A. ${ }^{2}$, SANTOS, J.B. ${ }^{3}$, FERREIRA, L.R. ${ }^{4}$, COSTA, M.D. ${ }^{5}$ e SILVA, A.A. ${ }^{4}$
}

\begin{abstract}
RESUMO - Neste trabalho, objetivou-se avaliar o efeito da microbiota associada à rizosfera de mucuna-preta (Stizolobium aterrimum) na fitorremediação de um solo contaminado pelo herbicida trifloxysulfuron-sodium. Os tratamentos no esquema fatorial $2 \times 4$ foram compostos por amostras de solo rizosférico de S. aterrimum cultivado por 60 dias e solo nãorizosférico ou não cultivado, todos submetidos a quatro níveis de contaminação pelo trifloxysulfuron-sodium $\left(0,0,7,5,37,5\right.$ e $\left.375 \mathrm{~g} \mathrm{ha}^{-1}\right)$. As amostras foram incubadas por 30 dias em recipientes apropriados para avaliação do desprendimento de dióxido de carbono $\left(\mathrm{CO}_{2}\right)$ pela respiração microbiana e quantificação de carbono da biomassa. Para comprovação de resultados, realizou-se o plantio de sorgo (Sorghum bicolor) nas amostras dos tratamentos, como planta indicadora da presença de resíduos do herbicida. Observou-se que o solo da rizosfera de $S$. aterrimum na presença do herbicida trifloxysulfuron-sodium se apresentou com maior atividade biológica, evidenciada pelo maior desprendimento de $\mathrm{CO}_{2}$ A biomassa microbiana foi maior nas amostras de solo rizosférico, independentemente da presença do herbicida. Comprovou-se a contribuição da microbiota no processo de descontaminação do solo, pelo fato de o crescimento das plantas de sorgo em solo rizosférico ter se apresentado semelhante na ausência ou presença do trifloxysulfuron-sodium, enquanto no solo nãorizosférico o crescimento do sorgo foi inversamente proporcional à dose do herbicida.
\end{abstract}

Palavras-chave: fitorremediação, descontaminação ambiental, leguminosa perene, mucuna-preta.

\begin{abstract}
This study aimed to evaluate the effect of microbiota associated to Stizolobium aterrimum rhizosphere on remediation of soil contaminated with trifloxysulfuron-sodium. The treatments were arranged in a factorial scheme $2 x 4$ and consisted of samples of Stizolobium aterrimum rhizosphere soils cultivated for 60 days and non-rhizosphere or non-cultivated soil, all submitted to four contamination levels $\left(0.0,7.5,37.5\right.$ and $\left.375 \mathrm{~g} \mathrm{ha}^{-1}\right)$.. Samples were incubated for 30 days in appropriate recipients to evaluate unfastening of $\mathrm{CO}_{2}$ by microbial respiration) and biomass carbon quantification. Bioremediation efficiency was verified by using the bioassay technique, using sorghum to indicate the presence of herbicide residue. The S. aterrimum rhizosphere soil treated with trifloxysulfuron-sodium showed higher biological activity, evidenced by higher unfastening of $\mathrm{CO}_{2}$. Rhizosphere soil samples showed higher microbial biomass, regardless of herbicide presence. It was concluded that microbiota contributed to soil decontamination, since sorghum growth on the rhizosphere soil was similar with or without trifloxysulfuron-sodium, while it was inversely proportional to the herbicide dose in the nonrhizosphere soil.
\end{abstract}

Keywords: remediation, environmental decontamination, perennial leguminous plants, S. aterrimum .

Recebido para publicação em 15.11.2006 e na forma revisada em 15.5.2007.

2 Estudante de Mestrado em Fitotecnia pela Universidade Federal de Viçosa, DFT/UFV. Av. P. H. Rolfs, s/n, Campus da UFV, 36570-000 Viçosa-MG < edsonapsant@yahoo.com.br >; ${ }^{3}$ Prof. de Agronomia da Faculdade de Ciências Agrárias, Universidade do Vale do Rio Doce - FAAG/UNIVALE, Governador Valadares-MG; ${ }^{4}$ Prof. Associado - Dep. de Fitotecnia - DFT/UFV; ${ }^{5}$ Prof. Adjunto do Dep. de Microbiologia - DMB/UFV. 


\section{INTRODUÇÃO}

A fitorremediação consiste na descontaminação de solo ou água utilizando-se plantas e sua microbiota associada. Essas espécies vegetais devem apresentar tolerância aos contaminantes e possuir habilidade de removê-los, extraí-los e/ou mineralizá-los no ambiente (Wilson et al., 2000). Atualmente a fitorremediação apresenta-se como alternativa em sistemas de cultivo que necessitam remover herbicidas de alta persistência no solo. Como exemplo, tem-se a descontaminação de áreas tratadas com o trifloxysulfuron-sodium na cultura do algodão, o qual pode apresentar problemas de carryover em culturas sensiveis plantadas até oito meses após a sua aplicação (Procópio et al., 2005).

As espécies vegetais utilizadas em programa de fitorremediação devem apresentar boa tolerância ao herbicida contaminante. Essa tolerância pode ser resultante de processos como a translocação diferencial de compostos orgânicos para outros fitotecidos, havendo subseqüente volatilização e/ou degradação parcial ou completa transformação em compostos menos tóxicos. Outra possibilidade é a fitoestimulação, na qual, em razão da liberação de exsudatos radiculares, há o estímulo à atividade microbiana, que atua degradando o composto contaminante no solo.

O principal mecanismo atuante na fitorremediação de agrotóxicos orgânicos não-clorados e herbicidas é a fitoestimulação ou rizodegradação (Pires et al., 2005). Esse mecanismo fitorremediador promove elevadas taxas de remediação, obtidas com utilização de plantas que, comprovadamente, aumentam a degradação microbiana de compostos orgânicos danosos ao solo (Anderson et al., 1993) e com diferenças significativas observadas, quando são comparados solos vegetados e não-vegetados (Siliciano \& Germida, 1999; Arthur et al., 2000).

Apesar de as propriedades físico-químicas da rizosfera apresentarem-se bem estáveis, a associação com o fornecimento constante de substratos orgânicos e fatores de crescimento favorece a intensa atividade metabólica e multiplicação das populações microbianas associadas, interferindo na capacidade destes em (co)metabolizar diferentes compostos presentes no meio. A interação entre raiz e solo promove a proliferação da comunidade microbiana na região da rizosfera, pela exsudação de nutrientes aminoácidos e polissacarídeos da planta (Arthur et al., 2000). O número de microrganismos no solo rizosférico é normalmente 5-10 vezes maior que naqueles nãorizosféricos, podendo chegar a mais de 100 vezes (Anderson et al., 1993). Portanto, a rizosfera apresenta potencial para degradação de xenobióticos, graças à comunidade microbiana.

Um fator muito importante no processo de rizodegradação é a capacidade de modificação do $\mathrm{pH}$ na região da rizosfera, influenciado pela eliminação de prótons e, principalmente, pelos exsudatos radiculares, pela absorção de nutrientes através do sistema radicular e por processos como a fixação biológica do $\mathrm{N}_{2}$ pela simbiose entre leguminosas e diazotróficos do solo. Modificações nos valores de $\mathrm{pH}$ podem tornar a remediação dos herbicidas mais acelerada. No caso do herbicida atrazine, verificou-se que sua mineralização aumentava rapidamente com o incremento do pH (Houot et al., 2000). Walker et al. (1996), estudando a degradação de isoproturon, diuron e metsulfuron-metil na solução do solo e na fração adsorvida em dois tipos de solo, concluíram que a meia-vida desses produtos foi menor em solução do solo onde a ação microbiana e a radicular foram maiores. Determinadas plantas estimulam a mineralização de alguns herbicidas, como atrazine e metolachlor, por meio do efeito rizosférico (Anderson et al., 1994; Anderson \& Coats, 1995; Perkovich et al., 1996; Burken \& Schnoor, 1996). Santos et al. (2006), avaliando diferentes espécies de adubos-verdes tolerantes ao trifloxysulfuron-sodium para fins de fitorremediação, verificaram que a espécie Stizolobium aterrimum se mostrou eficiente, sendo recomendada na descontaminação de solos tratados com esse herbicida.

Diante do exposto, objetivou-se com este trabalho avaliar a contribuição da microbiota as sociada à rizosfera da mucuna-preta (Stizolobium aterrimum) na fitorremediação de um solo contaminado com o herbicida trifloxysulfuron-sodium, com comprovação por meio do plantio do sorgo (Sorghum bicolor) como planta indicadora de resíduos do herbicida. 


\section{MATERIAL E MÉTODOS}

Inicialmente, foram cultivadas plantas de mucuna-preta (Stizolobium aterrimum) em vasos de $6,0 \mathrm{~kg}$ de substrato até ocorrer seu florescimento, o que se deu aos 60 dias após a emergência das plantas, bem como mantidos vasos somente com o mesmo substrato. No momento da colheita das plantas, foram retiradas amostras do solo aderido às raízes (rizosférico) e do solo sem cultivo (não-rizosférico). Os tratamentos no esquema fatorial $2 \times 4$ foram constituídos por dois tipos de solos (rizosférico e não-rizosférico), juntamente com quatro níveis de contaminação do substrato pelo herbicida trifloxysulfuron-sodium $(0,0,7,5,37,5 \mathrm{e}$ $375 \mathrm{~g} \mathrm{ha}^{-1}$ ), sendo, respectivamente, de 0,0 , $2,88,14,42$ e $144,23 \mu \mathrm{g} \mathrm{kg}^{-1}$ de herbicida por $\mathrm{kg}$ de solo. As doses do herbicida foram aplicadas nas amostras de solos por meio de uma microsseringa, sendo a calda distribuída uniformemente no solo, após elas terem sido passadas por peneira de 2 mesh, secas ao ar, determinado o teor de água, pesadas e acondicionadas em erlenmeyer. Após aplicação do herbicida, fez-se a incubação das amostras, mantendo-se o teor de água em torno de $70 \%$ da capacidade de campo. A respiração da microbiota do solo foi avaliada semanalmente, por meio da quantidade de $\mathrm{CO}_{2}$ evoluído durante 39 dias. O gás era capturado em frascos contendo $100 \mathrm{~mL}$ de $\mathrm{NaOH}$, com concentração de $0,25 \mathrm{~mol} \mathrm{~L}^{-1}$, em sistema contínuo de fluxo de ar isento de $\mathrm{CO}_{2}$ e umidade, sendo posteriormente titulado com $\mathrm{HCl}$ em $0,25 \mathrm{~mol} \mathrm{~L}^{-1}$, para quantificação.

Ao final do período de incubação, determinou-se o Carbono da Biomassa Microbiana (CBM) pelo método descrito por Vance et al. (1987), utilizando-se, em lugar do clorofórmio (fumigação), forno de microondas (irradiação) (Islam \& Weil, 1998). Com valores de $\mathrm{CO}_{2}$ evoluído e $\mathrm{CBM}$, determinou-se o quociente metabólico $\left(\mathrm{qCO}_{2}\right)$, fornecido pela relação entre o primeiro e o segundo, em $\mu \mathrm{g} \mu \mathrm{g}^{-1}$. Para omprovação dos resultados, nessas mesmas amostras cultivou-se o sorgo (Sorghum bicolor), espécie altamente suscetivel ao trifloxysulfuron-sodium, sendo indicadora da presença de resíduos do herbicida. Para isso, foram coletadas, de cada unidade experimental, subamostras de $100 \mathrm{~cm}^{3}$, as quais foram colocadas em vasos de $125 \mathrm{~cm}^{3}$, para cultivo da bioindicadora, por 14 dias. Avaliaram-se a altura média de plantas $(\mathrm{cm})$, a massa seca da parte aérea (g) e o nível de intoxicação visual (\%) recomendado por EWRC (1964).

Realizou-se análise de variância de todos os dados, cujas médias significativas foram ajustadas em curvas de regressão, relacionando doses de trifloxysulfuron-sodium e evolução de $\mathrm{CO}_{2}$ para cada tipo de solo (rizosférico e nãorizosférico), sendo os coeficientes testados pelo teste t a $5 \%$.

\section{RESULTADOS E DISCUSSÃO}

$\mathrm{Na}$ quantificação do $\mathrm{CO}_{2}$ evoluído, observou-se desprendimento maior desse gás sob ação da dose recomendada do herbicida de $7,5 \mathrm{~g} \mathrm{ha}^{-1}$, em comparação à dose 50 vezes maior (Figuras 1 e 2). Quando se compara o solo com cultivo ao não-cultivado, observouse maior atividade biológica no primeiro, evidenciada pela maior inclinação das curvas de regressão (Figuras 1 e 2). Apesar de grande parte dos herbicidas promover efeito negativo à microbiota do solo (Santos et al., 2006), verificou-se que a variação de disponibilidade e a possibilidade de transformação desses produtos permitem que determinados grupos de microrganismos sejam beneficiados (Edwards, 1989).

As maiores atividades biológicas no solo rizosférico ficaram em todos os niveis de contaminação do solo com o herbicida. A exsudação constante de nutrientes na interface solo-raiz promove a proliferação da população microbiana nessa região, permitindo o desenvolvimento de uma população microbiana muito superior àquela encontrada em ambiente nãorizosférico (Hsu \& Barth, 1979; Anderson et al., 1993; Arthur et al., 2000).

Na comparação da taxa de desprendimento do $\mathrm{CO}_{2}$ em relação às doses do herbicida para cada tipo de solo, observou-se que, na ausência do herbicida e no nível mais elevado de contaminação, a quantidade de $\mathrm{CO}_{2}$ desprendido foi menor comparada aos tratamentos que receberam as doses intermediárias do herbicida $\left(7,5\right.$ e $\left.37,5 \mathrm{~g} \mathrm{ha}^{-1}\right)$ (Tabela 1$)$. A elevada respiração basal do solo nem sempre está relacionada a efeito benéfico sobre os microrganismos. 


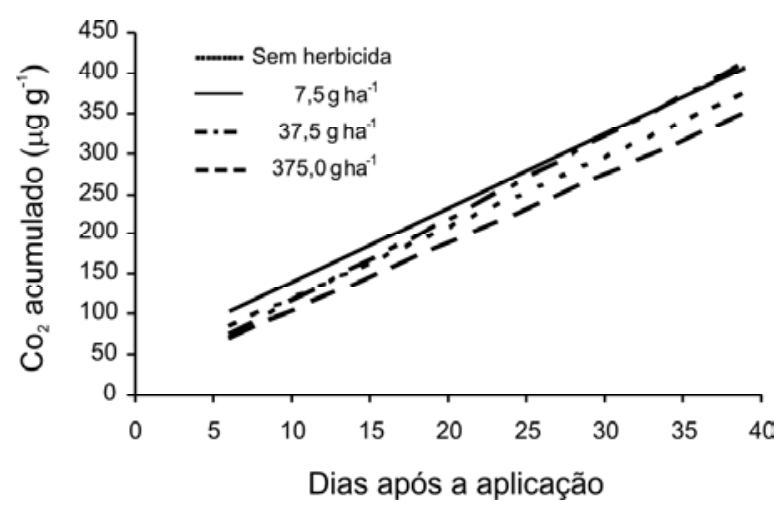

Figura 1 - Evolução de $\mathrm{CO}_{2}$ acumulado ao longo de 39 dias em solo proveniente da rizosfera de Stizolobium aterrimum e submetido a contaminações com o herbicida trifloxysulfuron-sodium $\left[0,0 \mathrm{~g} \mathrm{ha}^{-1}\right.$ (sem herbicida) ( $\left.\hat{\mathrm{Y}}=8,7866 \mathrm{x}+31,938 ; \mathrm{R}^{2}=0,99\right) ; 7,5 \mathrm{~g} \mathrm{ha}^{-1}$ $\left(\hat{Y}=9,2143 \mathrm{x}+47,879 ; \mathrm{R}^{2}=0,98\right) ; 37,5 \mathrm{~g} \mathrm{ha}^{-1}(\hat{\mathrm{Y}}=10,228 \mathrm{x}$ $\left.+14,169 ; \mathrm{R}^{2}=0,99\right)$; e $375,0 \mathrm{~g} \mathrm{ha}^{-1}(\hat{\mathrm{Y}}=8,4874 \mathrm{x}+19,325$; $\left.\left.\mathrm{R}^{2}=0,99\right)\right]$

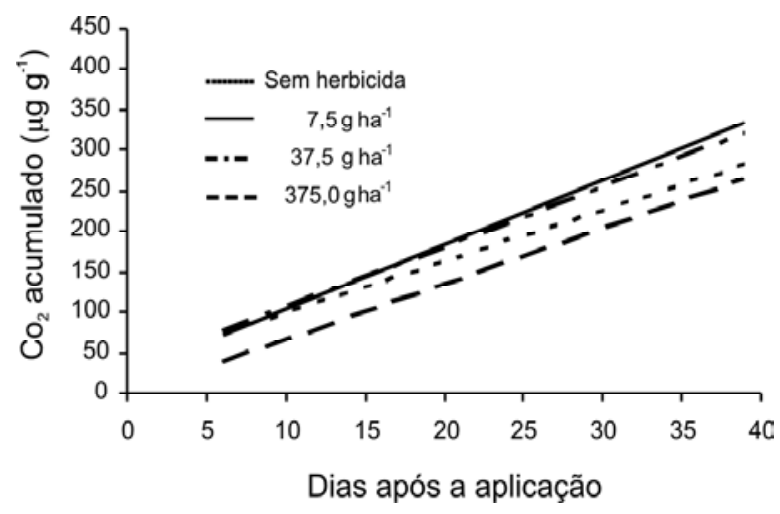

Figura 2 - Evolução de $\mathrm{CO}_{2}$ acumulado ao longo de 39 dias em solo sem cultivo prévio de Stizolobium aterrimum e submetido a contaminações com o herbicida trifloxysulfuron-sodium $\left[0,0 \mathrm{~g} \quad \mathrm{ha}^{-1} \quad\right.$ (sem herbicida) $\left(\hat{Y}=6,2482 \mathrm{x}+37,996 ; \mathrm{R}^{2}=0,97\right) ; 7,5 \mathrm{gha}^{-1}(\hat{\mathrm{Y}}=7,9459 \mathrm{x}+$ 23,$\left.872 ; \mathrm{R}^{2}=0,99\right) ; \quad 37,5 \mathrm{gha}^{-1} \quad(\hat{\mathrm{Y}}=7,3671 \mathrm{x}+33,506$; $\left.\mathrm{R}^{2}=0,98\right)$; e $\left.375,0 \mathrm{~g} \mathrm{ha}^{-1}\left(\hat{\mathrm{Y}}=6,8506 \mathrm{x}+1,5118 ; \mathrm{R}^{2}=0,97\right)\right]$.

Diversos fatores podem contribuir para mudanças no metabolismo microbiano, incluindo a degradação de compostos que podem ser usados como fonte de nutrientes (efeito benéfico), ou intoxicação por xenobióticos, que levam à menor eficiência de utilização do carbono (efeito maléfico), promovendo em ambos os casos aumento na emissão de $\mathrm{CO}_{2}$.
Os efeitos negativos de práticas agrícolas sobre a microbiota se verificam pela variação na biomassa microbiana. Observou-se que a biomassa de microrganismos foi maior para o solo proveniente da rizosfera da leguminosa, independentemente da aplicação do herbicida (Tabela 2). Os valores da biomassa microbiana no solo rizosfé rico variaram entre 298,08 e $347,46 \mu \mathrm{g} \mathrm{g}^{-1}$ de carbono, ao passo que no solo sem cultivo os valores permaneceram entre $177,62 \mu \mathrm{g} \mathrm{g}^{-1}$ de carbono, na ausência do trifloxysulfuron-sodium, e $266,50 \mathrm{\mu g} \mathrm{g}^{-1}$ de carbono, na dose de $37,5 \mathrm{~g}^{-1}{ }^{-1}$ do herbicida (Tabela 2). A variação na quantidade de microrganismos, observada no solo sem cultivo, entre as diferentes doses do herbicida pode estar relacionada ao fato de que, com o aumento na quantidade aplicada do produto, alguns grupos de microrganismos estariam utilizando-o como fonte de nutrientes, especialmente carbono, até um patamar de dose maior do produto, em que a intoxicação passa a ser maior do que a capacidade de degradação desse composto. A presença de moléculas tóxicas pode causar a morte de diversos grupos de microrganismos, os quais serviriam de substrato para o rápido crescimento de saprofiticos (Santos et al., 2006).

É provável que a diferença provocada na biomassa microbiana em função da presença do trifloxysulfuron no solo sem cultivo esteja relacionada ao fato de que nesse solo a diversidade em grupos de microrganismos que condiciona maior capacidade de resiliência esteja comprometida pela ausência de cultivos (Tótola \& Chaer, 2002). Dessa forma, o cultivo de S. aterrimum até seu completo florescimento favorece o acúmulo de exsudatos na região das raízes, permitindo crescimento e desenvolvimento de maior número de grupos de microrganis mos.

$\mathrm{O}$ quociente metabólico $\left(\mathrm{qCO}_{2}\right)$ estabelece a relação entre $\mathrm{o} \mathrm{CO}_{2}$ acumulado e o total da biomassa microbiana; com o aumento da eficiência dessa biomassa em utilizar os recursos disponíveis, menos carbono é perdido como $\mathrm{CO}_{2}$ pela respiração, podendo este ser incorporado aos tecidos microbianos (Anderson \& Domsch, 1985). O menor valor de $\mathrm{qCO}_{2}$ significa maior estabilidade da biomassa microbiana, equivalendo a maior estabilidade no sistema. $\mathrm{O}_{\mathrm{qCO}_{2}}$ pode indicar o equilibrio da microbiota 
Tabela 1 - Média diária de $\mathrm{CO}_{2}$ acumulado ao longo de 39 dias em solo proveniente ou não da rizosfera de Stizolobium aterrimum e submetido a contaminações com o herbicida trifloxysulfuron-sodium $\left(0,0 ; 7,5 ; 37,5 ;\right.$ e $\left.375,0 \mathrm{~g} \mathrm{ha}^{-1}\right)$

\begin{tabular}{|c|c|c|c|c|}
\hline \multirow{3}{*}{ Solo } & \multicolumn{4}{|c|}{ Doses de trifloxysulfuron-sodium $\left(\mathrm{g} \mathrm{ha}^{-1}\right)$} \\
\hline & 0,0 & 7,5 & 37,5 & 375,0 \\
\hline & \multicolumn{4}{|c|}{ 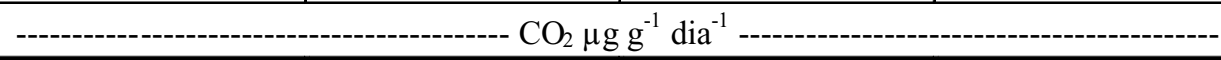 } \\
\hline Rizosférico & $9,58 \mathrm{ABa}$ & $10,29 \mathrm{Aa}$ & $10,22 \mathrm{Aa}$ & $8,85 \mathrm{Ba}$ \\
\hline Não-rizosférico & $7,10 \mathrm{ABb}$ & $8,49 \mathrm{Ab}$ & $7,95 \mathrm{ABb}$ & $6,60 \mathrm{Bb}$ \\
\hline
\end{tabular}

Médias seguidas por letras iguais, maiúsculas na linha, não diferem entre si pelo teste de Tukey a $5 \%$ de probabilidade, para c ada característica avaliada. Médias seguidas por letras iguais, minúsculas na coluna, não diferem entre si pelo teste $\mathrm{F}$ a $5 \%$ de $\mathrm{p}$ ro babilidade.

Tabela 2 - Valores para Carbono da Biomassa Microbiana (CBM) $\left(\mu \mathrm{g} \mathrm{g}^{-1}\right)$ em solo proveniente ou não da rizosfera de Stizolobium aterrimum, após aplicações de cinco doses do herbicida trifloxysulfuron-sodium $\left(0,0 ; 7,5 ; 37,5\right.$; e $\left.375 \mathrm{~g} \mathrm{ha}^{-1}\right)$ e incubação por 39 dias

\begin{tabular}{|c|c|c|c|c|}
\hline \multirow{3}{*}{ Solo } & \multicolumn{4}{|c|}{ Doses de trifloxysulfuron-sodium } \\
\hline & 0,0 & 7,5 & 37,5 & 375,0 \\
\hline & \multicolumn{4}{|c|}{ - } \\
\hline Rizosférico & $298,08 \mathrm{Aa}$ & $338,64 \mathrm{Aa}$ & $347,46 \mathrm{Aa}$ & $302,47 \mathrm{Aa}$ \\
\hline Não-rizosférico & $177,62 \mathrm{Bb}$ & $251,61 \mathrm{ABb}$ & $266,50 \mathrm{Aa}$ & $192,84 \mathrm{ABb}$ \\
\hline
\end{tabular}

Médias seguidas por letras iguais, maiúsculas na linha, não diferem entre si pelo teste de Tukey a 5\% de probabilidade, para cada característica avaliada. Médias seguidas por letras iguais, minúsculas na coluna, não diferem entre si pelo teste $\mathrm{F}$ a $5 \%$ de probabilidade.

do solo e a magnitude do distúrbio causado por determinada atividade agrícola (Santos et al., 2006). Apesar de não ter sido observada diferença significativa nos valores do $\mathrm{qCO}_{2}$ entre as doses de herbicida, o cultivo da leguminosa permitiu menor valor para esse coeficiente (Tabela 3). Como o $\mathrm{qCO}_{2}$ relaciona quantidade de $\mathrm{C}$ por unidade de biomassa microbiana, valores mais elevados evidenciam, comparativamente, menor capacidade de reaproveitamento do carbono no metabolismo microbiano. Acredita-se que, devido ao pequeno intervalo de tempo entre o plantio e a colheita da leguminosa (60 dias), não foi possivel encontrar efeito do trifloxysulfuron-sodium no $\mathrm{qCO}_{2}$. A aplicação de diferentes herbicidas ao longo do cultivo do feijoeiro promoveu aumento do $\mathrm{qCO}_{2}$, comparado aos tratamentos que não receberam os herbicidas (Santos et al., 2006). Verificou-se ainda que práticas culturais, como o plantio direto, diminuem consideravelmente o $\mathrm{qCO}_{2}$. Neste ensaio, a utilização do solo com o cultivo de leguminosa foi suficiente para promover diminuição no $\mathrm{qCO}_{2}$, ou seja, tornar esse ambiente menos instável do ponto de vista sustentável (Tabela 3). Acredita-se, também, que a capacidade já comprovada de S. aterrimum na remediação do trifloxysulfuronsodium (Procópio et al., 2004; Santos et al., 2006) possa ser resultante das interações direta e indireta com a microbiota atuante na região afetada pelas raízes.

A utilização do sorgo como espécie indicadora de resíduos do trifloxysulfuron-sodium foi eficiente na detecção do efeito desse herbicida. Nos tratamentos que receberam as doses superiores à comercial não houve desenvolvimento satisfatório das plantas de sorgo (Tabela 4). Apesar de a intoxicação, a altura e a massa seca do sorgo terem sido afetadas negativamente na presença do herbicida, ficou evidente o efeito amenizador do cultivo de $S$. aterrimum, visto que no solo que recebeu a dose de 7,5 $\mathrm{g} \mathrm{ha}^{-1}$ houve melhor desenvolvimento da planta indicadora (Tabela 4).

Pode-se concluir que a maior capacidade apresentada por $S$. aterrimum na descontaminação de solos com resíduo do trifloxysulfuronsodium envolve também o processo de 
Tabela 3 - Valores para o quociente metabólico $\left(\mathrm{qCO}_{2}\right)\left(\mu \mathrm{g} \mathrm{g}^{-1} \mathrm{dia}^{-1}\right.$ de $\mathrm{CO}_{2}$ por $\mu \mathrm{g} \mathrm{g} \mathrm{g}^{-1}$ de $\mathrm{C}$ da biomassa microbiana) em solo proveniente ou não da rizosfera de Stizolobium aterrimum, após aplicações de cinco doses do herbicida trifloxysulfuronsodium $\left(0,0 ; 7,5 ; 37,5 ;\right.$ e $\left.375 \mathrm{~g} \mathrm{ha}^{-1}\right)$ e incubação por 39 dias

\begin{tabular}{|c|c|c|c|c|c|}
\hline \multirow{3}{*}{ Solo } & \multicolumn{5}{|c|}{ Doses de trifloxysulfuron-sodium } \\
\hline & 0,0 & 7,5 & 37,5 & 375,0 & Média \\
\hline & \multicolumn{5}{|c|}{ - } \\
\hline Rizosférico & 0,032 & 0,030 & 0,029 & 0,029 & $0,030 \mathrm{a}$ \\
\hline Não- rizosférico & 0,040 & 0,034 & 0,030 & 0,034 & $0,035 b$ \\
\hline
\end{tabular}

Médias seguidas por letras iguais, minúsculas na coluna, não diferem entre si pelo teste $\mathrm{F}$ a $5 \%$ de probabilidade.

Tabela 4 - Efeitos de doses do herbicida trifloxysulfuron -sodium sobre a intoxicação visual (\%), altura (cm) e massa seca da parte aérea (MSPA, em g) de plantas de sorgo em solo proveniente ou não da rizosfera de Stizolobium aterrimum, aos 22 dias após a semeadura

\begin{tabular}{|c|c|c|c|c|}
\hline \multirow{3}{*}{ Solo } & \multicolumn{4}{|c|}{ Doses de trifloxysulfuron-sodium $\left(\mathrm{g} \mathrm{ha}^{-1}\right)$} \\
\hline & 0,0 & 7,5 & 37,5 & 375,0 \\
\hline & \multicolumn{4}{|c|}{----------------------------------------- Intoxicação visual (\%) ------------------------------------- } \\
\hline Rizosférico & $00 \mathrm{Ca}$ & $53 \mathrm{~B} \mathrm{a}$ & $88 \mathrm{~A} \mathrm{a}$ & $93 \mathrm{~A} \mathrm{a}$ \\
\hline Não-rizosférico & $00 \mathrm{~B} \mathrm{a}$ & $85 \mathrm{~A} \mathrm{a}$ & $88 \mathrm{~A} \mathrm{a}$ & $90 \mathrm{~A} \mathrm{a}$ \\
\hline \multirow[t]{2}{*}{$\mathrm{CV}(\%)$} & \multicolumn{4}{|c|}{ - } \\
\hline & \multicolumn{4}{|c|}{ - Altura $(\mathrm{cm})$ - } \\
\hline Rizosférico & $31,0 \mathrm{~A} \mathrm{a}$ & $16,3 \mathrm{~B} \mathrm{a}$ & $11,0 \mathrm{~B} \mathrm{a}$ & $3,0 \mathrm{C} \mathrm{a}$ \\
\hline Não-rizosférico & $30,3 \mathrm{~A} \mathrm{a}$ & $11,0 \mathrm{~B} \mathrm{~b}$ & $10,3 \mathrm{~B} \mathrm{a}$ & $3,7 \mathrm{C} \mathrm{a}$ \\
\hline \multirow[t]{2}{*}{$\mathrm{CV}(\%)$} & \multicolumn{4}{|c|}{-16, 142 - } \\
\hline & \multicolumn{4}{|c|}{ - } \\
\hline Rizosférico & $0,99 \mathrm{~A}$ a & $0,55 \mathrm{~B} \mathrm{a}$ & $0,39 \mathrm{Ca}$ & $0,05 \mathrm{Ca}$ \\
\hline Não-rizosférico & $1,06 \mathrm{~A} \mathrm{a}$ & $0,47 \mathrm{~B} \mathrm{a}$ & $0,05 \mathrm{C} \mathrm{b}$ & $0,01 \mathrm{C} \mathrm{a}$ \\
\hline
\end{tabular}

Médias seguidas por letras iguais, maiúsculas na linha, não diferem entre si pelo teste de Tukey a 5\% de probabilidade, para cada característica avaliada. Médias seguidas por letras iguais, minúsculas na coluna, não diferem entre si pelo teste $\mathrm{F}$ a $5 \%$ de probabilidade.

fitoestimulação. É provável que práticas agrícolas que favoreçam melhor desenvolvimento dessa leguminosa e o estabelecimento de diferentes grupos de microrganismos possibilitem maior eficiência no processo de fitorremediação do solo.

\section{AGRADECIMENTOS}

Ao Conselho Nacional de Desenvolvimento Científico e Tecnológico (CNPq), pelo apoio financeiro para realização deste trabalho.

\section{LITERATURA CITADA}

ANDERSON, T. A.; COATS, J. R. Screening rhizosphere soil samples for the ability to mineralize elevated concentrations of atrazine and metolachlor. J. Environ. Sci. Health, v. 30, p. 473-484, 1995.

ANDERSON, T. A.; KRUGER, E. L.; COATS, J. R. Enhanced degradation of a mixture of three herbicides in the rhizosphere of a herbicide-tolerant plant. Chemosphere, v. 28, p. 1551-1557, 1994.

ANDERSON, T. A.; GUTHRIE, E. A.; WALTON, B. T. Bioremediation in the rhizosphere: plant roots and associated microbes clean contaminated soil. Environ. Sci. Technol., v. 27, p. 2630-2636, 1993. 
ANDERSON, T. H.; DOMSCH, K. H. Determination of ecophysiological maintenance carbon requirements of soil microorganisms in a dormant state. Biol. Fertil. Soils, v. 1, p. 81-89, 1985 .

ARTHUR, E. L. et al. Degradation of an atrazine and metolachlor herbicide mixture in pesticide-contaminated soils from two agrochemical dealerships in Iowa. Water, Air, Soil Poll., v. 119, p. 75-90, 2000.

BURKEN, J. G.; SCHNOOR, J. L. Phytoremediation: plant uptake of atrazine and role of root exudates. J. Environ. Eng., v. 122, p. 958-963, 1996.

EDWARDS, C. A. Impact of herbicides on soils ecosystems. Crit. Rev. Plant Sci., v. 8, p. 221-257, 1989.

EUROPEAN WEED RESEARCH COUNCIL - EWRC. Report of 3 rd and $4 \mathrm{rd}$ meetings of EWRC. Cittee of methods in weed research. Weed Res., v. 4, p. 88, 1964.

HOUOT, S. et al. Dependence of accelerated degradation of atrazine on soil pH in French and Canadian soils. Soil Biol. Biochem., v. 32, p. 615-625, 2000.

HSU, T. S.; BARTHA, R. Accelerated mineralization of two organophos phate insecticides in the rhizosphere. Appl. Environ. Microbiol., v. 37 p. 36-41, 1979.

ISLAM, K. R.; WEIL, R. R. Microwave irradiation of soil for routine measurement of microbial biomass carbon. Biol. Fert. Soils, v. 27, p. 408-416, 1998.

PAUL, E. A.; CLARK, F. E. Carbon cycling and soil organic matter. In: PAUL, E. A.; CLARK, F. E. (Eds.). Soil microbiology and biochemistry. San Diego: Academic Press, 1996. p. 130-155.

PERKOVICH, B. S. et al. Enhanced mineralization of [ $\left.{ }^{14} \mathrm{C}\right]$ atrazine in $K$. scoparia rhizosferic soil from a pesticidecontaminated site. Pestic. Sci., v. 46, p. 391-396, 1996.

PIRES, F. R. et al. Inferências sobre atividade rizosférica de espécies com potencial para fitorremediação do herbicida tebuthiuron. R. Bras. Ci. Solo, v. 29, p. 627-634, 2005.
PROCÓPIO, S.O. et al. Seleção de plantas com potencial para fitorremediação de solos contaminados com o herbicida trifloxysulfuron-sodium. Planta Daninha, v. 22, n. 2, p. 315-322, 2004.

PROCÓPIO, S. O. et al. Fitorremediação de solo contaminado com trifloxysulfuron-sodium por mucuna-preta (Stizolobium aterrimum). Planta Daninha, v. 23, n. 4, p. 719-724, 2005.

RODRIGUES, B. N.; ALMEIDA, F. S. Guia de herbicidas. 5. ed. Londrina: Grafmarke, 2005. 591 p.

SANTOS J. B. Atividade microbiana após aplicação de herbicidas utilizados no cultivo do feijoeiro. 2005. $80 \mathrm{f}$. Tese (Doutora do em Fitotecnia) - Universidade Federal de Viçosa, Viçosa, 2005.

SANTOS, J. B. et al. Action of two herbicides on the microbial activity of soil cultivated with common bean (Phaseolus vulgaris) in conventional-till and no-till systems. Weed Res., v. 46, p. 284-289, 2006.

SCHINNER, F. et al. (Eds.) Indirect estimation of microbial biomass. In: Methods in soil biology. Heidelberg: SpringerVerlag, 1996 p. 47-75.

SILICIANO, S. D.; GERMIDA, J. J. Enhanced phyoremediation of chlorobenzoates in rhizosphere soil. Soil Biol. Biochem., v. 31, p. 299-305, 1999.

TÓTOLA, M. R.; CHAER, G. M. Microrganismos e processos microbiológicos como indicadores da qualidade dos solos. ALVAREZ V., V. H. et al. (Eds.). Tópicos em Ciência do Solo.Viçosa, MG: Sociedade Brasileira de Ciência do Solo, 2002. v.2.p. 195-276.

VANCE, E. D.; BROOKES, P. C.; JENKINSON, D. S. An extraction method for measuring soil microbial biomass C. Soil Biol. Biochem., v. 19, p. 703-707, 1987.

WALKER, A.; WELCH, S. J.; ROBERTS, S. J. Introduction and transfer of enhanced biodegradation of the herbicide napropamide in soils. Pest. Sci., v. 47, p.131-135, 1996.

WILSON, P. C.; WHITWELL, T.; KLAINE, S. J. Metalaxyl and simazine toxicity to and uptake by Typha latifolia. Arch. Environ. Contam. Toxicol., v. 39, p. 282-288, 2000. 